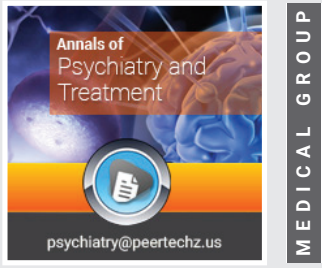

Research Article

\title{
Clinical evidence in the phenomenon of Demonic Possession
}

\section{Giulio Perrotta*}

Psychologist sp.ing in Strategic Psychotherapy, Forensic Criminologist, Legal Advisor sp.ed SSPL, Researcher, Essayist, Institute for the study of psychotherapies - ISP, Via San Martino della Battaglia no. 31,00185 , Rome, Italy

Received: 08 October, 2021

Accepted: 16 October, 2021

Published: 18 October, 2021

*Corresponding author: Dr. Giulio Perrotta, Psychologist sp.ing in Strategic Psychotherapy, Forensic Criminologist, Legal Advisor sp.ed SSPL, Researcher, Essayist, Institute for the study of psychotherapies - ISP, Via San Martino della Battaglia no. 31, 00185 , Rome, Italy, E-mail: info@giulioperrotta.com

ORCID: https://orcid.org/0000-0003-0229-5562

Keywords: Demons; Demonic possession; Possession; PICI-2; PDM-Q; PSM-Q; PAD-Q; PHEM

Copyright: () 2021 Perrotta G. This is an openaccess article distributed under the terms of the Creative Commons Attribution License, which permits unrestricted use, distribution, and reproduction in any medium, provided the original author and source are credited

https://www.peertechzpublications.com

\section{Check for updates}

\section{Abstract}

Purpose: Starting from the classic definition of "demonic possession" (as a psychophysical condition in which a person becomes the victim of a supernatural being of demonic origin), the present research, starting from the study published in 2019 on the proposed clinical classification of this particular phenomenon, is aimed at confirming the theoretical assumption of psychopathological origin, refuting the assumptions of the most significant analytical orientations, such as the ethnopsychiatric, the socio-anthropological, the cultural, the religious and the esoteric, to reaffirm the accuracy of the theoretical approach of the multifactorial model proposed in the previous research

Methods: Clinical interview, based on narrative-anamnestic and documentary evidence and the basis of the Perrotta Human Emotions Model (PHEM) concerning their emotional and perceptual-reactive experience, and administration of the battery of psychometric tests published in international scientific journals by the author of this work: 1) Perrotta Integrative Clinical Interviews (PICl-2), to investigate functional and dysfunctional personality traits; 2) Perrotta Individual Sexual Matrix Questionnaire (PSM-Q), to investigate the individual sexual matrix; 3) Perrotta Affective Dependence Questionnaire (PAD-Q), to investigate the profiles of affective and relational dependence; 4) Perrotta Human Defense Mechanisms Questionnaire (PDM-Q), to investigate the defence mechanisms of the Ego.

Results: The preliminary results of the interviews and the anamnestic form would suggest that the phenomenon of demonic possession has a greater tendency to manifest itself in the female group, in the juvenile group (and tends to decrease but not to disappear with the advancement of age) and in the group geographically originating in the centre-south of Italy (due to greater religious influences, popular beliefs and ancestral fideistic representations). Moreover, the subsequent findings would lead to deduce with almost total certainty, concerning the selected sample, that the phenomenon of demonic possession has an absolute prevalence in the believing population, faithful or in any case trusting in the existence of paranormal phenomena per se, even in the absence of objective and/or scientific evidence. Based on the $\mathrm{PICl}-2$ it emerged that the primary emerging disorder turns out to be alternatively the delusional disorder, the dissociative disorder and the obsessive disorder; followed, as secondary disorders, by the delusional disorder (if it is not considered as primary disorder), the schizoid disorder, the borderline disorder and the psychopathic disorder. Even the analysis of functional traits has reported the marked dysfunctional tendency of the classes that refer to self-control, sensitivity, Ego-ID comparison, emotionality, ego stability, security and relational functionality, reaffirming here too the marked dysfunctional tendency of the clinical population. According to the PSM-Q, more than $1 / 4$ of participants present a lack of acceptance of their sexual orientation and a marked tendency to chronicle feelings of shame into dysfunctional sexual behaviours of avoidance or hypersexuality. Still, nine in ten reports having experienced severe psychological or physical abuse at a young age, or intraparental relational imbalance, or otherwise a sexual upbringing that was not open and lacked free communication. According to the PDM-Q, $37.2 \%$ are affected by affective dependence, with a greater emphasis on types I (neurotic), V (borderline), III (histrionic), and VII (psychotic) in that order of descent. Finally, the PDM-Q reveals the widespread psychopathological tendency of the ego function framework for the mechanisms of isolation, denial, regression, reactive formation, denial, projection, removal, withdrawal, instinct, repression, and idealization.

Conclusions: The present research demonstrates beyond any reasonable doubt the psychopathological nature of the phenomenon of demonic possession, which deserves to be treated pharmacologically and with a psychotherapeutic approach (preferably cognitive-behavioural and/or strategic), according to the symptoms manifested and the severity of the morbid condition.

Citation: Perrotta G (2021) Clinical evidence in the phenomenon of Demonic Possession. Ann Psychiatry Treatm 5(1): 088-095. 


\section{Contents of the manuscript}

\section{Introduction and background}

In the literature, the theme of "demonic possession" is widely treated as a phenomenon of religious origin contaminated by social beliefs, personal interpretations and psychic impairments, but no study has ever demonstrated, with a representative sample, the real extent of the alleged psychopathological condition.

In 2019 [1], the writer tried to trace in specialized research all possible hypotheses that would explain the phenomena under consideration. Sifting, therefore, seven different approaches (ethnopsychiatric, cultural, socio-anthropological, esoteric, religious, psychoanalytic, clinical), in the previous research tried to demonstrate the theoretical inconsistency of the paranormal fabric and the evidence of the psychopathological condition according to the new "integrated approach" always identified by the writer, which identifies a precise scale of severity of the symptoms manifested by the patient:

1. Form I: possession as a manifestation of "substance intoxication disorder." Mystical experiences related to visions and auditory events of apparitions can be included in this category.

2. Form II: Possession as a manifestation of the "fictitious disorder". The patient, aware of playing a role, uses the possessive manifestation to draw attention to himself, according to the same pattern reproduced by patients with Munchausen Syndrome. The patient, in particular, may present different degrees of alteration of the real perception, where the self-produced fictitious symptom may even come to believe while remaining anchored to the fact that his need is always in attracting attention. Equivalent to the 2nd category of Ellenberger (latent possession).

3. Form III, type A: possession as a manifestation of "somatoform conversion disorder or conversion hysteria." The patient projects his or her deepest fears and anxieties through the possessive phenomenon, thereby reducing his or her responsibility for the original problem. In this form, moreover, the patient experiences a conversion of the deepest instances in a more radical, complex and chronic way, undergoing a modification of all or part of his personality. Egocentrism becomes the fixed pivot and his relationship with reality is compromised and deteriorated, resulting in the development of fantasy-type thoughts. May manifest obsessive-compulsive attitudes and/or manipulative, however, the hysteric does not pretend voluntarily because he does not realize the perceptual error; lives those circumstances as true and absolute. Moreover, if he is not noticed by others, his condition may be worsened, as the ultimate goal will always be to attract attention, albeit in a markedly more theatrical manner. We often see comorbidity with personality disorders: in this case, the form of possession takes on the characteristics of point 4). Ellenberger's 3rd category (spontaneous active possession) and cultural, sociological, and psychopathological positions fit perfectly into this framework.

4. Form III, type B: possession as a clinical manifestation of pathology of organic nature, such as "temporal lobe epileptic disorder", "parietal lobe syndrome" or even neoplastic brain diseases. These conditions can induce hallucinations capable of lucidly experiencing a possessive experience or even give the patient the feeling of being the victim of paranormal presences, through voices, sounds and frightening experiences. Mystical experiences fall under this "umbrella".

5. Form IV: possession as a manifestation of a "personality disorder" or "psychotic disorder". Examples may include possession related to "persecutory delusion" "psychosis" (not determined by drugs), "dissociation" and "schizophrenia". The patient has a well-defined personality disorder and possession is a symptom that recalls the disorder from which he or she is suffering. This is the framework of Ellenberger's 1st category (the severe psychic illness), ethno-anthropological positions, psychopathological positions, Séglas' classification and L. Gayral and J. Gayral's classification.

This theoretical approach appears, in the opinion of the writer, complete and detailed, and therefore in this research work will be used as a scale of evaluation concerning the symptoms manifested by patients, part of the selected population.

\section{Research objectives and methods}

Starting from the classic definition of "demonic possession" (as a psychophysical condition in which a person becomes the victim of a supernatural being of demonic origin), the present research, starting from the study published in 2019 on the proposed clinical classification of this particular phenomenon, is aimed at confirming the theoretical assumption of psychopathological origin, refuting the assumptions of the most significant analytical orientations, such as the ethnopsychiatric, the socio-anthropological, the cultural, the religious and the esoteric, to reaffirm the accuracy of the theoretical approach of the integrated multifactorial model.

The methods used are two: 1) Clinical interview, based on narrative-anamnestic and documentary evidence and the basis of the Perrotta Human Emotions Model (PHEM) concerning their emotional and perceptual-reactive experience; 2) Administration of the battery of psychometric tests published in international scientific journals by the author of this work: a) Perrotta Integrative Clinical Interviews (PICI-2), to investigate functional and dysfunctional personality traits; b) Perrotta Individual Sexual Matrix Questionnaire (PSM-Q), to investigate individual sexual matrix; c) Perrotta Affective Dependence Questionnaire (PAD-Q), to investigate affective and relational dependence profiles; d) Perrotta Human Defense Mechanisms Questionnaire (PDM-Q), to investigate ego defence mechanisms. 
The phases of the research were divided as follows:

1) Selection of the population sample, according to the parameters indicated in the following paragraph.

2) Clinical interview, to each population group.

3) Administration of the Perrotta Integrative Clinical Interviews (PICI-2), Perrotta Individual Sexual Matrix Questionnaire (PSM-Q), Perrotta Affective Dependence Questionnaire (PAD-O) e Perrotta Human Defense Mechanisms Questionnaire (PDM-O).

4) Data processing following administration.

5) Comparison of data obtained.

\section{Setting and participants}

The requirements decided for the selection of the sample population are:

1) Age between 18 years and 67 years, healthy and robust constitution and in the absence of pathological symptoms: this choice is oriented to exclude underage subjects because not identified during the research of the sample population and subjects of mature or elderly age to avoid possible implications with neurodegenerative medical conditions and/or neurovascular.

2) Italian nationality, with Italian ancestors in the last two generations: this choice is oriented in this sense to avoid cultural contaminations determined by popular beliefs different from the Christian-Catholic cult (such as, for example, South American religious cults that believe in the veracity of physical manifestations during states of alleged demonic possession).

3) Precocious and manifest physical and psychic symptoms attributable to the state of demonic possession (such as the mastery of languages not previously known or studied, knowledge of facts and remote episodes never disclosed or prediction of the future, extraordinary physical strength, aversion to the sacred and the images/ objects of religious worship, the genuine manifestation of paranormal phenomena such as telekinesis and significant alterations both physical and vocal able to interact with outer space modifying it with appropriate and calibrated instrumental surveys).

4) Declaration of state of possession on the basis of the subjective experience, in relation to the testimonies of family members, local ecclesiastical representatives intervened in the course of personal assistance and health personnel who found the dynamics reporting in the medical record psychiatric diagnosis related to the symptoms suffered by the patient (mainly disorders of the psychotic area).

5) Absence of acute state of demonic possession or another state of psychophysical alteration capable of justifying the symptomatic manifestation with other discomfort or disorder, both physical and psychic. In particular, to avoid false memories, memory lapses, or lack of cooperation from the patient, it was preferred to interview and administer the psychometric tests during the absence of an acute state of symptomatology.

The selected setting, taking into account the protracted pandemic period (already in progress since the beginning of the present research), is the online platform via Skype and Video call Whatsapp, both for the clinical interview and for the administration.

The present research work was carried out from March 2020 to October 2021. All participants were guaranteed anonymity and the ethical requirements of the Declaration of Helsinki are met.

Since the research is not financed by anyone, it is free of conflicts of interest.

The selected population clinical sample, which meets the requirements, is 323 participants, divided into five groups Tables 1-3.

\section{Results, limits and possible conflicts of in- terest}

After the selection of the chosen population sample (first stage), we proceeded with the clinical interviews (second stage), from which the first significant data emerged:

Table 1: Population sample (numerousness).

\begin{tabular}{|c|c|c|c|}
\hline Age & Male & Female & Total \\
\hline $18-27$ & 38 & 53 & 91 \\
\hline $28-37$ & 46 & 66 & 112 \\
\hline $38-47$ & 27 & 42 & 69 \\
\hline $48-57$ & 13 & 19 & 32 \\
\hline $58-67$ & 5 & 14 & 19 \\
\hline Total & $129(40 \%)$ & $194(60 \%)$ & $323(100 \%)$ \\
\hline
\end{tabular}

Table 2: Population sample (\% single category).

\begin{tabular}{|c|c|c|c|}
\hline Age & Male & Female & Total \\
\hline $18-27$ & $38(41.7 \%)$ & $53(58.3 \%)$ & $91(100 \%)$ \\
\hline $28-37$ & $46(41 \%)$ & $66(59 \%)$ & $112(100 \%)$ \\
\hline $38-47$ & $27(39.1 \%)$ & $42(60.9 \%)$ & $69(100 \%)$ \\
\hline $48-57$ & $13(40.6 \%)$ & $19(59.4 \%)$ & $32(100 \%)$ \\
\hline $58-67$ & $5(26.3 \%)$ & $14(73.7 \%)$ & $19(100 \%)$ \\
\hline
\end{tabular}

Table 3: Population sample (\% total sex).

\begin{tabular}{|c|c|c|}
\hline Age & Male & Female \\
\hline $18-27$ & $38(29.4 \%)$ & $53(27.3 \%)$ \\
\hline $28-37$ & $46(35.6 \%)$ & $66(34 \%)$ \\
\hline $38-47$ & $27(21 \%)$ & $42(21.6 \%)$ \\
\hline $48-57$ & $13(10.1 \%)$ & $19(9.8 \%)$ \\
\hline $58-67$ & $5(3.9 \%)$ & $14(7.3 \%)$ \\
\hline Total & $129(100 \%)$ & $194(100 \%)$ \\
\hline
\end{tabular}


1. Considering the total population sample (323/323), one immediately notices the good disparity between the populousness of the overall female sample (194) compared to the male sample (129), the former being $20 \%$ higher than the latter sample. Also noticeable is the higher populousness of 18 to 37-year-olds $(62.9 \%)$ compared to the cumulative 38-67-year-old range $(37.1 \%)$. Finally, the interviews show that the geographic origin of origin (family) is in $289 / 323$ (90\%) cases from central-southern Italy. These preliminary results would suggest that the phenomenon of demonic possession has a greater tendency to occur in the female group, in the youth and young adult group (and tends to decrease but not to disappear with age) and in the group geographically originating in the centre-south of Italy due to obvious greater religious influences, popular beliefs and ancestral fideistic representations.

2. Also during the first clinical interview, some data emerged in almost all cases, as if they explained the phenomenon more descriptively:

a) $100 \%$ of the population sample, in a cross-sectional way, without distinction of sexual gender or age, believe in a certain and dogmatic way the existence of paranormal phenomena, spiritual apparitions and demonic presences;

b) $100 \%$ of the population sample, across the board, without distinction of sexual gender or age, reports having experienced extrasensory and/or paranormal experiences [2-4], since a very young age;

c) $100 \%$ of the population sample, across the board, without distinction of sexual gender or age, reports believing in Christianity as a primary religious current, of which $317 / 323(98.1 \%)$ of cases declare themselves to be "non-practising Catholics" without, however, knowing all the rules and dogmas of the Church to which they adhere and without having read in-depth the sacred text of reference; the remaining 5/323 (1.6\%) declare themselves to have a different faith, but always related to the Christian current, while $1 / 323$ (0.3\%) declare themselves to be Muslim. Again, $100 \%$ of the population sample reports having at least one ascendant family member who is a frequent attendee of religious cults and ceremonies, a position in turn inherited from a parent.

d) $100 \%$ of the population sample, transversally, without distinction of sexual gender or age, report to have felt the first symptoms consistent with a picture of presumed demonic possession within the first 18 years, but to have matured the loss of control and the subjection to the spiritual entity only in later and adult age. In 320/323 of the cases (99.1\%), the first hysterical, dissociative and/ or delusional episode concerning the phenomenological condition (mistaken for demonic possession) occurred after the age of 12 , while in the remaining $3 / 323(0.9 \%)$ of the cases it occurred in preschool and childhood. e) $100 \%$ of the population sample, across the board, without distinction of sexual gender or age, appears significantly hyperactive and therefore the administration of the battery of questionnaires appears relevant and functional for the analysis.

f) $92.6 \%(299 / 323)$ presented recurrent gastrointestinal symptoms (with a clear prevalence of constipation, related to fermentative dysbiosis) treated pharmacologically or with homoeopathic or pharmacy products, without complete remission of symptoms $[5,6]$.

3. Using, during the interview, the strategic language $[7,8]$ and the Perrotta Human Emotions Model (PHEM) [9], it emerged that the totality of the selected population sample presents a full distress orientation, facilitating feelings such as guilt, shame, anger, fear and disappointment, in the presence of past (childhood) and current (interpersonal and work) family traumas [10-14]. In fact, without the administration of questionnaires, it is evident that the impairment of perception in the plane of reality [15-17] involves per se the manifestation of paranormal episodes described by patients, in the presence of a probable personality disorder to be identified with the help of psychodiagnostic tools.

The third stage of the research focused on the administration of the battery of questionnaires and these revealed the following results:

1. Administration of the Perrotta Integrative Clinical Interviews (PICI-2) [18-23]: Concerning the analysis of the dysfunctional traits (PICI-2TA), the primary disorder that emerged with at least 5 traits is delusional disorder $(178 / 323$ or $55.1 \%)$, dissociative disorder $(88 / 323$ or $27.2 \%$ ) and obsessive disorder (39/323 or $12 \%)$; This is followed, as secondary disorders, by delusional disorder (if not considered as a primary disorder, 190/323 or $58.8 \%$ ), schizoid disorder (63/323 or $19.5 \%$ ), borderline disorder ( $48 / 323$ or $14.9 \%)$ and psychopathic disorder $(16 / 323$ or $5 \%)$. A separate category that deserves specific mention is schizophrenic disorder, which was first diagnosed in $2 / 323(0.6 \%)$ of the population sample (1 woman and 1 man, both in the 18-27 age group) after administration of the PICI-2, confirmed by the subsequently proposed psychiatric examination. In 307/323 (95.7\%), the following disorders emerged as comorbidities: ADHD (in the population under 37 years of age), body dysmorphism, ICT disorder, sleep disorders, nutrition disorders, anxiety and mood disorders, paraphiliac disorders, and behavioural addictions (especially technology and internet addiction). The concrete suicidal risk emerged in $7 / 323$ cases $(2.2 \%)$, while the presumed risk or demand for attention emerged in $203 / 323$ (62.8\%). Equally interesting is the cause/concause "epilepsy": 56/323 (17.3\%) report being under antiepileptic therapy, while 196/323 (60.7\%) report having had in their lives at least one episode referable to epilepsy and/or syncopal state of vasovagal 
origin. Another significant finding emerges from the correlation between the condition suffered and the massive use of body tattoos: $58 / 323$ or $18 \%$ have tattoos on more than $15 \%$ of the body. On the other hand, in the analysis of functional traits (PICI-2FT), it emerged that the classes most compromised because they tended to be dysfunctional (with values of 0 or 4 ) were those referring to self-control, sensitivity, action, Ego-ID comparison, emotionality, ego stability, security and relational functionality, reiterating here too the marked dysfunctional tendency of the clinical population [2458]. The preference for administering the PICI-2 over other widely validated and used psychometric tests, such as the MMPI-2, was for reasons of expediency: in fact, previous research has demonstrated the effectiveness and efficiency, sometimes better indicated, of the PICI-2 over the MMPI-2, in terms of performance and completeness of diagnosis $[20,23]$.

2. Administration of the Perrotta Individual Sexual Matrix Questionnaire (PSM-Q) [59,60]: The PSM questionnaires demonstrated that $86 / 323(26.6 \%)$, equally in both the male and female samples, have a lack of acceptance of their sexual orientation and a marked tendency to chronicle feelings of shame into dysfunctional sexual avoidance or hypersexual conduct. Furthermore, $287 / 323(88.9 \%)$ state that they have experienced serious psychological or physical abuse at a young age, or an intraparental relational imbalance, or in any case a sexual upbringing that was not open and lacking in free communication. Concerning dysfunctional psychophysical sexual conditions, 291/323 (90.1\%) declared themselves to be sexually dissatisfied because they were suffering from a sexual pathology; circumstances that emerged later and were confirmed by questionnaire $\mathrm{C}$ and questionnaire $\mathrm{D}$, as well as by the test on dysfunctional sexual behaviour (in the latter case, with scores above 30/50). On the other hand, questionnaires A and B on sexual relational style revealed in $232 / 323(71.8 \%)$ a polygamous tendency that was, in any case, dysfunctional, sublimated into monogamy but tending towards omission and betrayal (the latter with scores above 28/50 and 38/75).

3. Administration of the Perrotta Affective Dependence Questionnaire (PAD-Q) [61,62]: Administration of the questionnaire confirmed the finding that emerged indirectly during the administration of the PICI-2, specifying the weights involved: $9 / 129(7 \%)$ for the male sample and $111 / 194$ (57.2\%) for the female sample had a pathological score higher than 95/175 (54.3\%), for an overall total score of $120 / 323(37.2 \%)$, with a greater accentuation of types I (neurotic), V (borderline), III (histrionic) and VII (psychotic) in this order of decreasing, demonstrating on the one hand that the condition of affective dependence is less marked and indicative of PICI values but is at the same time relevant in over $1 / 3$ of the total sample.
4. Administration of the Perrotta Human Defense Mechanisms Questionnaire (PDM-Q) [63,64]: The administration of the questionnaire reported the following data: in $285 / 323(88.2 \%)$ values of 4 and 5 were found on the mechanisms of isolation, denial, regression, reactive formation, denial, projection, removal, withdrawal, instinct, repression and idealization, confirming the widespread psychopathological tendency of the framework of ego function.

\section{Conclusions}

The last two steps served to reorder the results, and then draw conclusions. In particular:

1. Results obtained by Clinical interview: The preliminary results of the interviews and the anamnestic form would suggest that the phenomenon of demonic possession has a greater tendency to occur in the female group, in the juvenile group (and tends to decrease but not to disappear with the advancement of age) and in the group geographically originating in the centre-south of Italy (for obvious greater religious influences, popular beliefs and ancestral fideistic representations). Moreover, the following results would lead to deduce with almost total certainty, concerning the selected sample, that the phenomenon of demonic possession has an absolute prevalence in the believing population, faithful or in any case trusting in the existence of paranormal phenomena per se, even in the absence of objective and/or scientific evidence. $92.6 \%(299 / 323)$ present recurrent gastrointestinal symptoms (with a clear prevalence of constipation, related to fermentative dysbiosis) treated pharmacologically or with homoeopathic or pharmacy products, without complete remission of the symptoms.

2. Results were obtained by Perrotta Integrative Clinical Interviews (PICI-2): The primary emerging disorder turns out to be alternatively the delusional disorder, the dissociative disorder and the obsessive disorder; followed, as secondary disorders, by the delusional disorder (if it is not considered as primary disorder), the schizoid disorder, the borderline disorder and the psychopathic disorder. A separate category that deserves specific mention is schizophrenic disorder, which was first diagnosed in two cases after administration of the PICI-2. Even the analysis of functional traits have reported the marked dysfunctional tendency of the classes that refer to self-control, sensitivity, acting out, Ego-ID comparison, emotionality, ego stability, security and relational functionality, reaffirming here too the marked dysfunctional tendency of the clinical population.

3. Results were obtained by Perrotta Individual Sexual Matrix Questionnaire (PSM-Q): The PSM questionnaires showed that more than a quarter of the participants present a lack of acceptance of their sexual orientation and a marked tendency to chronicle the feeling of shame in dysfunctional sexual behaviours of avoidance or 
hypersexuality. Furthermore, nine out of ten states that they have experienced serious psychological or physical abuse at a young age, or an intraparental relational imbalance, or in any case a sexual upbringing that was not open and lacking in free communication.

4. Results were obtained by Perrotta Affective Dependence Questionnaire (PAD-Q): The administration of the questionnaire confirmed the data emerged indirectly during the administration of the PICI-2, specifying the weights involved: $9 / 129(7 \%)$ for the male sample and $111 / 194(57.2 \%)$ for the female sample had a pathological score above 95/175 (54.3\%), for an overall total score of $120 / 323(37.2 \%)$, with a greater accentuation of types I (neurotic), V (borderline), III (histrionic) and VII (psychotic) in this order of descent, demonstrating on the one hand that the condition of affective dependence is less marked and indicative of the PICI values but is at the same time relevant in over $1 / 3$ of the total sample.

5. Results were obtained by Perrotta Human Defense Mechanisms Questionnaire (PDM-Q): The administration of the questionnaire reported the widespread psychopathological tendency of the ego function framework for the mechanisms of isolation, denial, regression, reactive formation, denial, projection, removal, withdrawal, instinct, repression and idealization.

Based on these results, resuming the classification proposed in the previous research, it is possible to draw a precise comparative scale with the selected sample of the population Table 4 .

In conclusion, this research demonstrates beyond reasonable doubt the psychopathological nature of the phenomenon of demonic possession, which deserves to be treated pharmacologically and with a psychotherapeutic approach (preferably cognitive-behavioural and/or strategic) [65], according to the symptomatology manifested and the severity of the morbid condition.

Table 4: Perrotta Demonic Possession Scale (PDPS).

\begin{tabular}{|c|c|c|c|c|c|c|}
\hline Pathological form & \multicolumn{3}{|c|}{ No. } & \multicolumn{3}{|c|}{$\%$} \\
\hline Form I & $6 / 129$ & $18 / 194$ & $24 / 323$ & $4,6 \%$ & $9,3 \%$ & $7,4 \%$ \\
\hline Form II & $14 / 129$ & $27 / 194$ & $41 / 323$ & $10,8 \%$ & $13,9 \%$ & $12,7 \%$ \\
\hline Form III/A & $21 / 129$ & $35 / 194$ & $56 / 323$ & $16,3 \%$ & $18 \%$ & $17,3 \%$ \\
\hline Form III/B & $30 / 129$ & $26 / 194$ & $56 / 323$ & $23,3 \%$ & $13,4 \%$ & $17,4 \%$ \\
\hline Form IV & $58 / 129$ & $88 / 194$ & $146 / 323$ & $45 \%$ & $45,4 \%$ & $45,2 \%$ \\
\hline Total & $129 / 129$ & $194 / 194$ & $323 / 323$ & $100 \%$ & $100 \%$ & $100 \%$ \\
\hline
\end{tabular}

\section{References}

1. Perrotta G (2019) The phenomenon of demoniac possession: definition, contexts and multidisciplinary approaches. J Psychology and Mental Health Care 3: 1-019. Link: https://bit.ly/3aMwYPb

2. Perrotta G (2019) The Pineal Gland: anatomical, clinical and neurobiochemical profiles, between hypotheses of the past, certainties of the present and future perspectives. J Neurol Psychol 7: 5. Link: https://bit.ly/2Txh9ql

3. Perrotta G (2019) The neural correlates in the presumed extrasensory faculties of the medium and in the perception on the sacred. J Neurol Psychol 7: 07. Link: https://bit.ly/3iGYQaT

4. Perrotta G (2020) Alien Abduction Experience: definition, neurobiological profiles, clinical contexts and therapeutic approaches. Ann Psychiatry Treatm 4: 025-029. Link: https://bit.ly/3kNOSHq

5. Perrotta G (2021) The intestinal microbiota: towards a multifactorial integrative model. Eubiosis and dysbiosis in morbid physical and psychological conditions. Arch Clin Gastroenterol 7: 024-035. Link: https://bit.ly/36VwRPC

6. Perrotta G (2021) Intestinal dysbiosis: definition, clinical implications, and proposed treatment protocol (Perrotta Protocol for Clinical Management of Intestinal Dysbiosis, PID) for the management and resolution of persistent or chronic dysbiosis. Arch Clin Gastroenterol 7: 056-063. Link: https://bit.ly/3BVKrQH

7. Perrotta G (2020) The strategic clinical model in psychotherapy: theoretical and practical profiles. J Addi Adol Behav 3: 5. Link: https://bit.ly/3aPMx9X

8. Perrotta G (2020) Accepting "change" in psychotherapy: from consciousness to awareness. Journal of Addiction Research and Adolescent Behaviour 3. Link: https://bit.ly/36Vw80Q

9. Perrotta G (2021) The "Human Emotions" and the "Perrotta Human Emotions Model" (PHEM): The new theoretical model. Historical, neurobiological and clinical profiles. Arch Depress Anxiety 7: 020-027. Link: https://bit.ly/2XkVTWY

10. Perrotta G (2020) Psychological trauma: definition, clinical contexts, neural correlations and therapeutic approaches. Curr Res Psychiatry Brain Disord: CRPBD-100006. Link: https://bit.ly/37UD3bz

11. Perrotta G (2020) Dysfunctional attachment and psychopathological outcomes in childhood and adulthood. Open J Trauma 4: 012-021. Link: https://bit.ly/2Mi2ThB

12. Perrotta G (2020) Neonatal and infantile abuse in a family setting. Open $\mathrm{J}$ Pediatr Child Health 5: 034-042. Link: https://bit.ly/2KApVQo

13. Perrotta G (2021) Parental Alienation Syndrome (PAS): definition, humanistic profiles and clinical hypothesis of absorption with "adaptation disorder". Clinical evidence. Open J Pediatr Child Health 6: 026-035. Link: https://bit.ly/2XngdqT

14. Perrotta G, Fabiano G (2021) Behavioural disorders in children and adolescents: Definition, clinical contexts, neurobiological profiles and clinical treatments. Open J Pediatr Child Health 6: 005-015. Link: https://bit.ly/3DPRYBg

15. Perrotta G (2019) The reality plan and the subjective construction of one's perception: the strategic theoretical model among sensations, perceptions, defence mechanisms, needs, personal constructs, beliefs system, social influences and systematic errors. J Clinical Research and Reports 1. Link: https://bit.ly/3b34baH

16. Perrotta G (2019) Delusions, paranoia and hallucinations: definitions, differences, clinical contexts and therapeutic approaches. Cientific Journal of Neurology (CJNE) 1: 22-28. Link: https://bit.ly/3ht2nKz

17. Perrotta G (2021) The state of consciousness: from perceptual alterations to dissociative forms. Defining, neurobiological and clinical profiles. J Neuro Neurol Sci Disord 7: 006-018. Link: https://bit.ly/3n24oPI

18. Perrotta G (2020) The structural and functional concepts of personality: The new Integrative Psychodynamic Model (IPM), the new Psychodiagnostic Investigation Model (PIM) and the two clinical interviews for the analysis of personality disorders (Perrotta Integrative Clinical Interview or PICI) for adults and teenagers (1TA version) and children (1C version), Psychiatry Peertechz, E-book. Link: https://bit.ly/2SqQevV 
19. Perrotta G (2020) First revision of the Psychodiagnostic Investigation Model (PIM-1R) and elaboration proposal of a clinical interview for the analysis of personality disorders (Perrotta Integrative Clinical Interview or $\mathrm{PICl}-1)$ for adults, teenagers and children, Psychiatry Peertechz. Link: https://bit.ly/2MQe3dY

20. Perrotta G (2020) "Perrotta Integrative Clinical Interview (PICl-1)": Psychodiagnostic evidence and clinical profiles in relation to the MMPI-II, Ann Psychiatry Treatm 4: 062-069. Link: https://bit.ly/3q0bYLP

21. Perrotta G (2021) "Perrotta Integrative Clinical Interview" (PICI) for adults and teenagers (1TA version) and children (1C version): new theoretical models and practical integrations between the clinical and psychodynamic approach. Ann Psychiatry Treatm 5: 001-014. Link: https://bit.ly/3546iGM

22. Perrotta G (2021) Perrotta Integrative Clinical Interview (PICl-1): a new revision proposal for PICl-1TA. Two single cases. Glob J Medical Clin Case Rep 8: 041 049. Link: https://bit.ly/3rtXLaq

23. Perrotta G (2021) Perrotta Integrative Clinical Interviews (PICl-2): innovations to the first model, the study on the new modality of personological investigation, trait diagnosis and state diagnosis, and the analysis of functional and dysfunctional personality traits. An integrated study of the dynamic behavioural, cognitive and constructivist models in psychopathological diagnosis. Ann Psychiatry Treatm 5: 067-083. Link: https://bit.ly/3DTK2yC

24. Perrotta G (2019) Anxiety disorders: definitions, contexts, neural correlates and strategic therapy. J Neur Neurosci 6: 046. Link: https://bit.ly/2WSmiaT

25. Perrotta G (2019) Neural correlates in eating disorders: Definition, contexts and clinical strategies. J Pub Health Catalog 2: 137-148. Link: https://bit.ly/3mWmf8s

26. Perrotta G (2019) Post-traumatic stress disorder: Definition, contexts, neural correlations and cognitive-behavioral therapy. J Pub Health Catalog 2: 40-47. Link: https://bit.ly/3rvaCc6

27. Perrotta G (2019) Sleep-wake disorders: Definition, contexts and neura correlations. J Neurol Psychol 7: 09. Link: https://bit.ly/3hoBiGO

28. Perrotta G (2019) Tic disorder: definition, clinical contexts, differential diagnosis, neural correlates and therapeutic approaches. J Neurosci Rehab 2019: 1-6. Link: https://bit.ly/36UJme5

29. Perrotta G (2019) Depressive disorders: Definitions, contexts, differential diagnosis, neural correlates and clinical strategies. Arch Depress Anxiety 5 : 009-033. Link: https://bit.ly/2KADvDm

30. Perrotta G (2019) Panic disorder: definitions, contexts, neural correlates and clinical strategies. Current Trends in Clinical \& Medical Sciences 1. Link: https://bit.ly/38IG6D5

31. Perrotta G (2019) Obsessive-Compulsive Disorder: definition, contexts, neural correlates and clinical strategies. Cientific Journal of Neurology 1: 08-16. Link: https://bit.ly/3pxNbNu

32. Perrotta G (2019) Behavioral addiction disorder: definition, classifications, clinical contexts, neural correlates and clinical strategies. J Addi Adol Beh 2. Link: https://bit.ly/3rAT9ip

33. Perrotta G (2019) Paraphilic disorder: definition, contexts and clinical strategies. J Neuro Research 1: 4. Link: https://bit.ly/3gxr1t3

34. Perrotta G (2019) Internet gaming disorder in young people and adolescent: a narrative review. J Addi Adol Beh 2.

35. Perrotta G (2019) Bipolar disorder: definition, differential diagnosis, clinical contexts and therapeutic approaches. J Neuroscience and Neurological Surgery 5. Link: https://bit.ly/34SoC67

36. Perrotta G (2020) Suicidal risk: definition, contexts, differential diagnosis, neural correlates and clinical strategies. J Neuroscience Neurological Surgery 6: 114. Link: https://bit.ly/3aMqcu5
37. Perrotta G (2020) Pathological gambling in adolescents and adults: definition, clinical contexts, differential diagnosis, neural correlates and therapeutic approaches. ES J Neurol 1: 1004. Link: https://bit.ly/3rT9H5A

38. Perrotta G (2020) Pedophilia: definition, classifications, criminological and neurobiological profiles and clinical treatments. A complete review. Open J Pediatr Child Health 5: 019-026. Link: https://bit.ly/38Jzggz

39. Perrotta G (2020) The concept of altered perception in "body dysmorphic disorder": the subtle border between the abuse of selfies in social networks and cosmetic surgery, between socially accepted dysfunctionality and the pathological condition. J Neurol Neurol Sci Disord 6: 001-007. Link: https://bit.ly/3uWvlHv

40. Perrotta G (2020) Sexual orientations: a critical review of psychological, clinical and neurobiological profiles. Clinical hypothesis of homosexual and bisexual positions. Int $\mathrm{J}$ Sex Reprod Health Care 3: 027-041. Link: https://bit.ly/38DtEVa

41. Perrotta G (2020) Borderline Personality Disorder: definition, differential diagnosis, clinical contexts and therapeutic approaches. Ann Psychiatry Treatm 4: 043-056. Link: https://bit.ly/3hx2B1N

42. Perrotta G (2020) Narcissism and psychopathological profiles: definitions, clinical contexts, neurobiological aspects and clinical treatments. J Clin Cases Rep 4: 12-25. Link: https://bit.ly/2X8wzzF

43. Perrotta G (2020) Dysfunctional sexual behaviours: definition, clinical contexts, neurobiological profiles and treatments. Int J Sex Reprod Health Care, 3(1) 061-069, DOI: 10.17352/ijsrhc.000015.

44. Perrotta G (2020) Bisexuality: definition, humanistic profiles, neural correlates and clinical hypotheses. J Neuroscience and Neurological Surgery 6. Link: https://bit.ly/2L6VXmA

45. Perrotta G (2021) Histrionic personality disorder: Definition, clinical profiles, differential diagnosis and therapeutic framework. Arch Community Med Public Health 7: 001-005. Link: https://bit.ly/3cugaOH

46. Perrotta G (2020) Affective Dependence: from pathological affectivity to personality disorders. Definitions, clinical contexts, neurobiological profiles and clinical treatments. Health Sci 1: 1-7. Link: https://bit.ly/2TXmTdj

47. Perrotta G (2020) Psychotic spectrum disorders: definitions, classifications, neural correlates and clinical profiles. Ann Psychiatry Treatm 4: 070-084. Link: https://bit.ly/2QI9kNc

48. Perrotta G (2021) Maladaptive stress: Theoretical, neurobiological and clinical profiles. Arch Depress Anxiety 7: 001-007. Link: https://bit.ly/3sDs39Y

49. Perrotta G (2021) Sexual fantasies: the boundary between physiology and psychopathology. Int J Sex Reprod Health Care 4: 042-052. Link: https://bit.ly/3hYo31x

50. Perrotta G (2021) Clinical evidence in sexual orientations: definitions, neurobiological profiles and psychological implications. Ann Psychiatry Treatm 5: 043-053. Link: https://bit.ly/3y3QWz8

51. Perrotta G (2021) "Polygamous perception" and couple's relational choice: definitions, socio-cultural contexts, psychopathological profiles and therapeutic orientations. Clinical evidence. Ann Psychiatry Treatm 5: 054-061. Link: https://bit.ly/3aMCQbf

52. Perrotta G (2021) The learning of specific dysfunctional behavioural patterns through social network and telematics platforms in preadolescents and adolescents. Psychopathological clinical evidence. Open J Pediatr Child Health 6: 026-035. Link: https://bit.ly/30CYD3I

53. Perrotta G (2020) Epilepsy: from pediatric to adulthood. Definition, classifications, neurobiological profiles and clinical treatments. J Neurol Neurol Sci Disord 6: 014-029. Link: https://bit.ly/3vz3ltv

54. Perrotta G (2020) The pharmacological treatment of epileptic seizures in

Citation: Perrotta G (2021) Clinical evidence in the phenomenon of Demonic Possession. Ann Psychiatry Treatm 5(1): 088-095

DOI: https://dx.doi.org/10.17352/apt.000035 
children and adults: introduction, clinical contexts, psychopharmacological profiles and prospects in the neurogenetic field. Journal of Neuroscience and Neurological Surgery 6. Link: https://bit.ly/3g3hRmV

55. Perrotta G (2021) Avoidant personality disorder: Definition, clinical and neurobiological profiles, differential diagnosis and therapeutic framework. $J$ Neuro Neurol Sci Disord 7: 001-005. Link: https://bit.ly/3DPRF9A

56. Perrotta G (2021) Clinical evidence in Troilism (Polygamy and Polyamory): definition, psychological profiles and clinical implications. Int J Sex Reprod Health Care 4: 073-079. Link: https://bit.ly/3vmgyq6

57. Perrotta G (2021) The diagnosis of personality traits in "affective dependency": when the toxic bond is an expression of a personality disorder. Research. Int $J$ Sex Reprod Health Care 4: 085-090. Link: https://bit.ly/3ja3nUq

58. Perrotta G (2021) Massive use of tattoos and psychopathological clinical evidence. Arch Community Med Public Health 7: 079-085. Link: https://bit.ly/3eMoKcu

59. Perrotta G (2021) Perrotta Individual Sexual Matrix Questionnaire (PSM-1). The new clinical questionnaire to investigate the main areas of the individual sexual matrix. Int J Sex Reprod Health Care 4: 013-021. Link: https://bit.ly/3irnlof
60. Perrotta G (2021) "Perrotta Individual Sexual Matrix Questionnaire" (PSM-Q) Technical updates and clinical research. Int J Sex Reprod Health Care 4: 062 066. Link: https://bit.ly/3iqhJA0

61. Perrotta G (2021) Perrotta Affective Dependence Questionnaire (PAD-Q) Clinical framing of the affective-sentimental relational maladaptive model. Ann Psychiatry Treatm 5: 062-066. Link: https://bit.ly/3aJs9Gx

62. Perrotta G (2021) Perrotta Affective Dependence Questionnaire (PAD-Q) Psychodiagnostic evidence and clinical profiles. Int J Sex Reprod Health Care 4: 080-084. Link: https://bit.ly/2YX5MLX

63. Perrotta G (2020) Human mechanisms of psychological defence: definition, historical and psychodynamic contexts, classifications and clinical profiles. Int J Neurorehabilitation Eng 7: 1. Link: https://bit.ly/2LOI5d」

64. Perrotta G (2021) "Perrotta Human Defense Mechanisms Questionnaire" (PDM-Q): The new psychodiagnostic tool to identify human psychological defense mechanisms and their clinical implications. Arch Depress Anxiety 7 : 029-033. Link: https://bit.ly/3jcsYw2

65. Perrotta G (2021) Strategic psychotherapy and the "decagonal model" in clinical practice. Ann Psychiatry Treatm 5: 028-035. Link: https://bit.ly/3iCCwzs
Discover a bigger Impact and Visibility of your article publication with Peertechz Publications

\section{Highlights}

* Signatory publisher of ORCID

* Signatory Publisher of DORA (San Francisco Declaration on Research Assessment)

* Articles archived in worlds' renowned service providers such as Portico, CNKI, AGRIS, TDNet, Base (Bielefeld University Library), CrossRef, Scilit, J-Gate etc.

* Journals indexed in ICMJE, SHERPA/ROMEO, Google Scholar etc.

* OAI-PMH (Open Archives Initiative Protocol for Metadata Harvesting)

* Dedicated Editorial Board for every journal

* Accurate and rapid peer-review process

* Increased citations of published articles through promotions

* Reduced timeline for article publication

Submit your articles and experience a new surge in publication services (https://www.peertechz.com/submission).

Peertechz journals wishes everlasting success in your every endeavours. 